\title{
BMJ Open Respiratory function and respiratory complications in spinal cord injury: protocol for a prospective, multicentre cohort study in high-income countries
}

\author{
Anja M Raab (D) , ${ }^{1}$ Martin W G Brinkhof (D) , ${ }^{2}$ David J Berlowitz (D) ,3,4 \\ Karin Postma (i) , ${ }^{5}$ David Gobets (D) , ${ }^{6}$ Sven Hirschfeld, ${ }^{7}$ Maria T E Hopman (D) , \\ Burkhart Huber, ${ }^{9}$ Margret Hund-Georgiadis (D) , ${ }^{10}$ Xavier Jordan (D) , ${ }^{11}$ \\ Martin Schubert, ${ }^{12}$ Renate Wildburger (D) ${ }^{13}$ Gabi Mueller (D) ${ }^{1}$
}

To cite: Raab AM, Brinkhof MWG, Berlowitz DJ, et al. Respiratory function and respiratory complications in spinal cord injury: protocol for a prospective, multicentre cohort study in high-income countries. BMJ Open 2020;10:e038204. doi:10.1136/ bmjopen-2020-038204

- Prepublication history for this paper is available online. To view these files, please visit the journal online (http://dx.doi. org/10.1136/bmjopen-2020038204).

AMR and MWGB contributed equally.

Received 11 March 2020 Revised 16 July 2020 Accepted 05 0ctober 2020

Check for updates

(C) Author(s) (or their employer(s)) 2020. Re-use permitted under CC BY-NC. No commercial re-use. See rights and permissions. Published by BMJ.

For numbered affiliations see end of article.

Correspondence to

Dr Gabi Mueller;

gabi.mueller@paraplegie.ch

\section{ABSTRACT}

Introduction Pneumonia is one of the leading complications and causes of death after a spinal cord injury (SCI). After a cervical or thoracic lesion, impairment of the respiratory muscles decreases respiratory function, which increases the risk of respiratory complications. Pneumonia substantially reduces patient's quality of life, may prolong inpatient rehabilitation time, increase healthcare costs or at worse, lead to early death. Respiratory function and coughing can be improved through various interventions after $\mathrm{SCl}$, but the available evidence as to which aspect of respiratory care should be optimised is inconclusive. Furthermore, ability of respiratory function parameters to predict pneumonia risk is insufficiently established. This paper details the protocol for a large-scale, multicentre research project that aims to evaluate the ability of parameters of respiratory function to predict and understand variation in inpatient risk of pneumonia in SCl. Methods and analysis RESCOM, a prospective cohort study, began recruitment in October 2016 across $10 \mathrm{SCl}$ rehabilitation centres from Australia, Austria, Germany, the Netherlands and Switzerland. Inpatients with acute SCl, with complete or incomplete cervical or thoracic lesions, 18 years or older and not/no more dependent on 24-hour mechanical ventilation within the first 3 months after injury are eligible for inclusion. The target sample size is 500 participants. The primary outcome is an occurrence of pneumonia; secondary outcomes include pneumonia-related mortality and quality of life. We will use the longitudinal data for prognostic models on inpatient pneumonia risk factors.

Ethics and dissemination The study has been reviewed and approved by all local ethics committees of all participating centres. Study results will be disseminated to the scientific community through peer-reviewed journals and conference presentations, to the $\mathrm{SCl}$ community, other stakeholders and via social media, newsletters and engagement activities.

Trial registration details ClinicalTrials.gov NCT02891096.

\section{INTRODUCTION}

Pneumonia is a leading complication and cause of death after a spinal cord injury (SCI),
Strengths and limitations of this study

- RESCOM is the first multinational study to prospectively evaluate predictors of pneumonia from a representative sample of persons with spinal cord injury (SCI) receiving inpatient rehabilitation in a high-income setting.

- The RESCOM cohort will enrol 500 persons with SCI to develop generalisable prognostic models as well as to validate causal risk factors, specifically impaired respiratory function, of pneumonia risk.

- Because respiratory function following $\mathrm{SCl}$ may be improved through respiratory muscle training, study results may inform and improve current clinical practice and patient management through the better targeting of interventions.

- Generalisability of the study with respect to pneumonia risk is limited to patients with less than 24 hours of mechanical ventilation within the first 3 months after injury, because respiratory function cannot be measured using standard techniques in those who are intubated.

- This study will provide insight whether the improvement of respiratory muscle strength and respiratory function represent promising targets for intervention to reduce pneumonia risk following $\mathrm{SCl}$.

even in high-income countries. ${ }^{12}$ In newly injured patients, pneumonia may substantially complicate and lengthen the period of first rehabilitation, while community dwelling persons living with SCI are commonly re-hospitalised for pneumonia over extended periods that frequently involve intensive care. $^{34}$

Contemporary healthcare policy and patient management aim to improve healthrelated Quality of Life (QoL) and life expectancy in the SCI community as well as to reduce infection-related healthcare costs. Reducing the incidence of pneumonia is, 
therefore, a major objective. Critical to this goal is that (1) persons with an elevated risk of pneumonia can be identified early (prediction), (2) modifiable risk factors are known (causality) and readily measurable and (3) effective interventions targeting key risk factors are established. Unfortunately, however, the contemporary evidence base regarding risk groups and modifiable risk factors of pneumonia in SCI, and subsequent effective intervention strategies, remain scant. ${ }^{5-8}$ Most existing studies evaluating between-person differences in risk of pulmonary complications assessed non-modifiable factors only: demographics (sex and age), injury severity (level and completeness) or spinal shock severity ${ }^{568}$ Only one recent study investigated modifiable risk factors for pneumonia, that is, steroid administration, which may be helpful to maintain muscle mass and strength but may also cause other relevant side effects. ${ }^{9}$

Impairment of respiratory function represents the most promising target for clinically relevant research on pneumonia in SCI, as these measures are directly linked to the neurological impairment and appear to be modifiable through targeted respiratory training. ${ }^{10-12}$ Respiratory muscles below the level of injury may become paralysed or impaired ${ }^{13}$ and respiratory function is compromised with higher levels of injury causing greater impairment. ${ }^{10} 14$ Cough impairment is also considered critically important, as insufficient removal of airway secretions may result in the development of mucus plugging and complications such as atelectasis or pneumonia. ${ }^{15} 16$ Effective coughing comprises an inspiration, compression and expulsion phase. Cough impairment following SCI may affect each phase due to the weakening of inspiratory and expiratory muscle function, which may decrease the maximum volume of expelled air by restricting both the maximum inspiratory volume prior to contraction as well as a reduction in the amount able to be expelled. ${ }^{15} 17$ The limited evidence in SCI suggests that inspiratory (using maximal inspiratory muscle pressure, $\mathrm{PI}_{\max }$ ) rather than expiratory function (maximal expiratory muscle pressure, $\mathrm{PE}_{\max }$ ) is the prime determinant of cough capacity (peak flow), particularly in patients with a motor-complete cervical SCI. ${ }^{18}$ Postma et al found that impaired pulmonary function may increase respiratory infections at 1 year after SCI, but their study did incorporate that respiratory muscle strengthening and respiratory complications were incompletely assessed. ${ }^{7}$

To the best of our knowledge, there is currently no comprehensive database available for the further development of generalisable prognostic models, nor to improve causal inference of pneumonia risk in light of impairment in respiratory function. The multicentre and multinational cohort study, RESpiratory COMplications (RESCOM), aims to establish such an evidence base in SCI. We believe RESCOM will thereby improve clinical practice through better targeting of interventions during the inpatient setting in high-income countries.

\section{METHODS AND ANALYSES \\ Design and setting}

RESCOM is a prospective international, multicentre cohort study in high-income countries. Data collection commenced in October 2016 across 10 specialised rehabilitation centres for SCI from Austria (2 centres), Australia (1 centre), Germany (1 centre), the Netherlands (2 centres) and Switzerland (4 centres), and is still ongoing.

\section{Study population}

Newly injured persons who are aged 18 years or older, admitted for inpatient rehabilitation in the participating centres, with complete or incomplete lesions (grades A-D on the American Spinal Injury Association (ASIA) Impairment Scale (AIS) $)^{19}$ and cervical or thoracic lesion levels (right and left motor levels between C1 and T12) are eligible for study participation. Persons with severe pre-existing scoliosis, progressive neurological diseases, 24-hour mechanical ventilation dependency until more than 3 months after injury or severe mental disorders are excluded.

\section{Sample size}

For the analysis of pneumonia risk we estimated, over a conservative range of pneumonia event probabilities from 0.1 to 0.2 , the minimal sample size needed to detect a plausible HR (effect size of interest) of 1.7 or more for inspiratory muscle strength (principal predictor variable) ${ }^{20}$ Using a conventional power of 0.8 and significance level of 0.05 , this analysis indicated a sample size of 500 as adequate for the purpose of the present study.

\section{Procedures}

The measurement schedule of RESCOM includes up to four measurement time-points (T1-T4) during the inpatient rehabilitation period (figure 1). Following start of rehabilitation, newly injured patients are contacted for

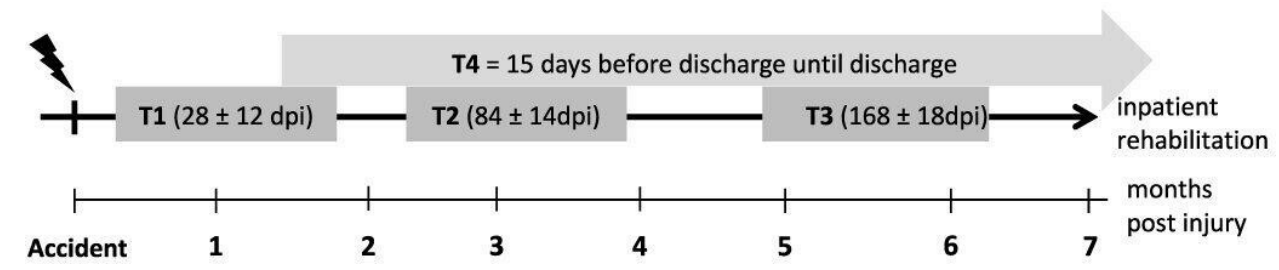

Figure 1 Time frames for measurements (time-ponts: T1-T4) during inpatient rehabilitation. T1-T3 time-windows with days post injury (dpi) are shown by grey bars and T4 by the grey arrow above. 
recruitment at about 4 weeks (T1) or at 12 weeks (T2) if the first 6 weeks or more weeks following injury were spend in the intensive care unit (ICU) or in a general hospital for acute care. Subsequent assessments are planned at 24 weeks (T3) and at discharge to the community (T4). The $\mathrm{T} 4$ time-point may precede and replace T2 and/or T3 in patients with a shorter length of inpatient rehabilitation stay. Temporal start and length of inpatient stay for rehabilitation varies with injury severity, general health status of patients and between countries and clinics. Consequently, between two and four measurement time-points are anticipated across patients, with those with more severe lesions and the longest length of stay providing more time points. In the four Swiss centres, RESCOM is run as a 'nested project' of the Swiss SCI (SwiSCI) cohort study (www.swisci.ch). ${ }^{20}$ The temporal schedule of data collection of RESCOM is aligned to that of SwiSCI and the relevant data for RESCOM will be extracted from the SwiSCI database additionally to RESCOM-specific measurements and questionnaires.

\section{Quality control}

Each of the participating centres has one or two responsible study nurses. Before the start of recruitment, a study nurse meeting in the Swiss Paraplegic Centre Nottwil was performed to train all study nurses for the procedures and the assessments of the study. A study manual has been established to give an overview of the procedures and all assessed variables. A frequently asked question sheet is available in the study-specific database (secuTrial, iAS, Berlin, Germany) with all relevant questions the study nurses asked during data collection. For quality control of data assessment, regular central (database secuTrial) and one local monitoring visit in each of the participating centres are performed. The coordinating study centre supports one central study coordinator who is responsible for central and local monitoring as well as for support of the local study nurses for questions concerning patient inclusion, data collection and entry into the database.

\section{Parameters assessed \\ Primary outcome}

The occurrence of pneumonia is the primary outcome of RESCOM, observed continuously during the whole risk period of interest from time of injury until the end of inpatient rehabilitation. Medical records of inpatients are screened for diagnosis of pneumonia, including records from the acute care phase before admission to the rehabilitation centre. Pneumonia is classified by type and cause, and the date of onset and duration of each event is recorded. Pneumonia is clinically diagnosed using the criteria described in the pneumonia flow diagram as endorsed by the Centers for Disease Control and Prevention. ${ }^{21}$ Mortality is defined as pneumonia related, if pneumonia was clinically recorded as the initiating cause of events leading to death. Other causes of death are similarly classified.

\section{Participant characteristics}

All participant characteristics are obtained from medical records. Basic characteristics that are collected at $\mathrm{T} 1$ include gender, age, height, cause of SCI (traumatic or non-traumatic) and smoking history. Parameters that may temporally vary, including body weight, motor lesion level and AIS, medication, frequency of defecation as well as medical complications, are assessed on all available measurement time-points. At T4, the actual smoking status and International Classification of Diseases (ICD10) coded comorbidities are recorded additionally.

\section{Additional parameters}

Additional parameters assessed at all measurement timepoints, that is, up to four times per participant (further details on these measurements and questionnaires are given below):

- Measurement of respiratory muscle strength and lung function.

- International Spinal Cord Society (ISCoS) Pulmonary Function Data Set. ${ }^{22}$

- Quantiative questionnaire on physical exercise training.

- Quantitative questionnaire on respiratory therapy and respiratory muscle training.

- Bogenhausener Dysphagia Score (BODS).

- ISCoS QoL Questionnaire. ${ }^{23}$

- COVID-19 assessment form.

\section{Measurement of respiratory function}

Measurement of respiratory function consists of respiratory muscle strength ( $\mathrm{PI}_{\max }$ and $\left.\mathrm{PE}_{\max }\right)$ and lung function with forced vital capacity (FVC), forced expiratory volume in $1 \mathrm{~s}\left(\mathrm{FEV}_{1}\right)$, peak expiratory flow $(\mathrm{PEF})$ and peak cough flow (PCF). All measurements are performed at the same day according to the American Thoracic Society/European Respiratory Society guidelines ${ }^{24}$ in a sitting position either in the participant's own wheelchair or on a chair in which participants are able to walk. To derive a reliable estimate of the highest value for each parameter, each measurement is repeated until the three highest values of a given parameter are within a $20 \%$ range. The highest value of each parameter is retained for further analysis. ${ }^{25}$

Measurement of respiratory muscle strength and lung function have been harmonised across the 10 centres using identical equipment. $\mathrm{PI}_{\max }$ and $\mathrm{PE}_{\max }$ are measured using a hand-held respiratory pressure metre (Micro RPM, Micro Medical, Hoechberg, Germany). The $\mathrm{PI}_{\text {max }}$ measurement is derived from residual volume and $\mathrm{PE}_{\max }$ from total lung capacity, against the occluded one-way valve of the respiratory pressure metre with the pressure maintained for at least $1 \mathrm{~s}$. To derive the maximum pressure over a 1-second period, the patient is instructed to maintain inspiratory and expiratory pressure for at least $1.5 \mathrm{s.}^{26}$ Abdominal binders are removed prior to any measurement of respiratory function. ${ }^{27}$

The FVC is the total volume of air the participant is able to exhale after a maximal inspiration. The $\mathrm{FEV}_{1}$ is 
the total volume of air that has been exhaled at the end of the first second of maximal forced expiration. PEF is the maximum flow of air achieved during the maximum expiratory flow manoeuvre. ${ }^{28}$ During the PCF manoeuvre, the maximum flow of air is measured by having the participant cough as forcefully as possible through a peak flow metre. Participants breathe through a mouthpiece while wearing a nose clip. Across study centres, lung function parameters are measured accordingly, but using three different brands of portable spirometer, including Micro Loop (Care Fusion, Basingstoke, the UK; all Swiss, Dutch and German centres), EasyOne Spirometer (Niche Medical, Melbourne, Australia; Australian centre), Masterscreen PFT Pro (Care Fusion, Hoechberg, Germany; one Austrian centre) and Vitalograph (Ennis, Ireland; one Austrian centre).

\section{ISCOS Pulmonary Function Data Set}

The ISCoS Pulmonary Function Data Set ${ }^{22}$ consists of questions on pulmonary complications (asthma, chronic obstructive pulmonary disease, sleep apnoea and others) before and after SCI, smoking history, current utilisation of pulmonary assistance and lung function measurement.

\section{Questionnaires on physical exercise and respiratory muscle} training as well as on respiratory therapy

The questionnaires on individual respiratory muscle training, regular physical exercise and therapy are kept as simple as possible. Only quantitative or yes/no questions on physical activities performed during the last 7 days are asked. Physical activity, duration of sport activities as well as number of physiotherapy sessions per week are recorded. Respiratory therapies such as mobilisation of secretions, manual blowing or air stacking and insufflation/exsufflation are assessed on a yes/no basis and if yes, whether with or without manual cough assistance. Respiratory muscle training is assessed separately for inspiratory and expiratory muscle strength training as well as respiratory muscle endurance training (ie, isocapnic hyperpnoea). In case of training, the name of the device, the number of training sessions per week, the number of repetitions per session as well as the resistance is noted.

\section{Bogenhausener Dysphagia Score}

Dysphagia is assessed using the BODS, which consist of two scales, each with a score from 1 to 8 , resulting in a sum score of 2-16. The first scale quantifies swallowing of saliva and whether the patient has a tracheal cannula. For patients with tracheal cannula, the degree of blocking is quantified as fully, partly or mainly not blocked. The second scale quantifies problems with oral ingestion, including 4 of the 8 scores for parenteral nutrition. The BODS is assessed by a speech therapist or a physiotherapist, in close coordination with the RESCOM study nurse.

\section{ISCoS Quality of Life questionnaire}

QoL is evaluated using the ISCoS QoL questionnaire. ${ }^{23}$ This measurement instrument accepts a multifaceted concept and includes three questions that capture general
QoL (overall well-being), rating of physical health and satisfaction with psychological health.

\section{COVID-19 assessment form}

At the start of the COVID-19 pandemic (March 2020), we implemented an additional form into the study database and instructed all study nurses of each participating centre to additionally fill in this form for the actually included and future patients. Since COVID-19 infections are often leading to severe pneumonia and probably also death, we identified this as a potential confounder of our study results and, therefore, have to include this infection into our analysis. The COVID-19 form includes information on diagnosis, date of diagnosis, symptoms and death due to COVID-19.

\section{Database secuTrial}

To enable secure capture and management of RESCOM data, the professional and web-browser-based database system secuTrial is used. SecuTrial fulfils the minimal requirements for data storage and management indicated in the good clinical practice guidelines and also supports the central monitoring of data collection across all participating centres. Database set-up, personal accounts with pre-defined roles for all study collaborators as well as support, data export and archiving are provided by a study-independent database manager from the studycentre in Nottwil, Switzerland.

\section{Methods of minimising bias}

Study participants receive an introduction for the study procedure by the local study nurse skilled in the management of persons with SCI and trained for all study specific tasks. A standardised study protocol was defined to minimise attrition bias.

The coding of the participants is conducted by the study nurses of each site in order to keep the data management and the biostatistician blinded (de-identified at source). The coding list remains with the study nurses of each site for the whole duration of the study and archiving period. Thus, coding is conducted without any influence of the principal investigator, the data manager or biostatistician. The study investigators strive for complete separation of the persons involved in the steps of enrolment and data collection from those involved in the data management and analysis.

All assessments are conducted and entered into the study database by the trained study nurse(s) of each participating centre.

\section{Patient and public involvement}

No patients or public have been directly involved in the planning or conduct of this study. Study results will be disseminated to the scientific community through peerreviewed journals and conference presentations, to the SCI community, other stakeholders and via social media, newsletters and engagement activities. 


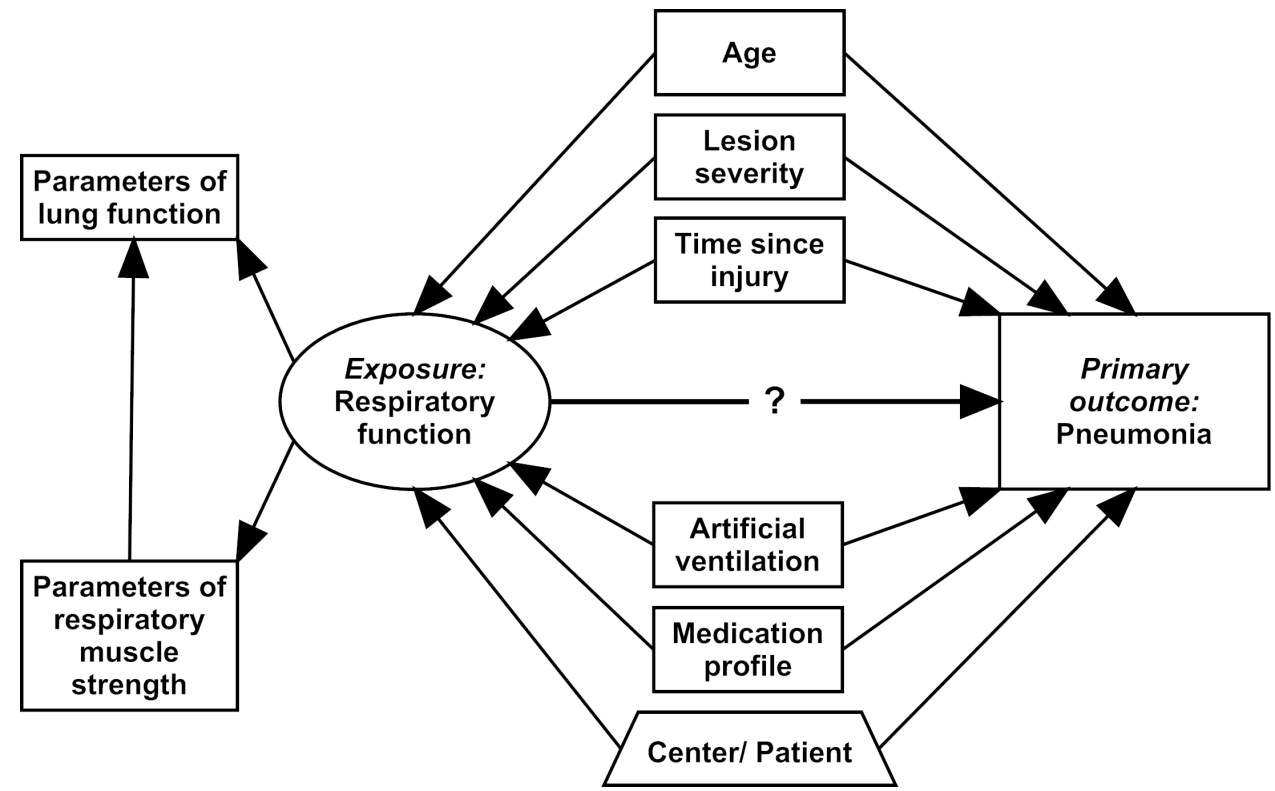

Figure 2 Global scheme of the planned data analysis investigating the dynamic association between respiratory function and pneumonia. In regression modelling, all variables will be time-updated in accordance with the repeated measurement schedule of RESpiratory COMplications and controlling for between-person and between-centre sources of variance. Respiratory function is operationalised using parameters of respiratory muscle strength and parameters of lung function.

\section{Statistics}

Statistical analysis will involve basic descriptive statistics and multivariable regression analyses that take into account the longitudinal and multilevel structure of the data. A global overview of the multivariable data analysis plan for investigating the association between respiratory function and pneumonia is given in figure 2 . To evaluate variation in pneumonia risk, time-to-event analysis techniques (eg, flexible parametric survival modelling) will be employed, taking date of injury as the starting point of the risk period. Regression models targeting causal inference will make use of time-updated information for all variables in accordance with the repeated measurement schedule of RESCOM, while controlling for betweenperson and between-centre sources of variance using random and fixed effects. The exposure 'respiratory function' will be operationalised using parameters of respiratory muscle strength and parameters of lung function, as a latent construct within generalised latent variable modelling or as individual parameters in selected analysis as required. ${ }^{29}$ Critical confounders that have been defined using evidence from the literature and expert opinion of RESCOM collaborators include age, lesion severity, time since injury (structurally captured in timeto-event modelling), artificial ventilation and medication. Models targeting prognosis of pneumonia during first rehabilitation will only include parameters of respiratory function that have been collected at baseline..$^{30}$

\section{ETHICS AND DISSEMINATION}

Full ethical approval for this project has been obtained by all local ethics committees of all participating centres. We certify that all applicable institutional and governmental regulations concerning the ethical use of data of human volunteers are followed during the course of this research.

Potential protocol modifications and amendments will be submitted to the ethical committees for approval. This project has been registered with ClinicalTrials.gov NCT02891096 and regular study updates are presented on Research Gate (https://www.researchgate.net/ project/RESCOM-RESpiratory-COMplications-afterSpinal-Cord-Injury).

All participants gave written or witnessed verbal consent, if upper limb function is too impaired for a participant to sign, prior to entering the study.

The study results will be disseminated through publication in scientific journals, presentation at relevant conferences, directly to the participants and clinicians as well as on social media and in newsletters. The dissemination aims to provide clinicians with reliable prognostic factors to identify persons who are at heightened risk of pneumonia and thus, to effectively reduce pneumonia risk and pneumonia-related hospitalisations.

\section{STRENGTH AND LIMITATIONS}

RESCOM is the first prospective, international study that reports modifiable predictors of pneumonia from a representative sample of persons with SCI during inpatient rehabilitation. Its comprehensive evidence base facilitates the systematic evaluation of the discriminatory power of respiratory function parameters for pneumonia risk in high-income countries.

The RESCOM study is effective in recruiting a representative sample of inpatients with a motor complete or incomplete SCI in Austria, Australia, Germany, the Netherlands and Switzerland. The anticipated minimal sample 
size of 500 should provide project sufficient statistical power to answer the key hypothesis that respiratory function, and $\mathrm{PI}_{\max }$ in particular, is a strong prognostic parameter that quantifies clinical pneumonia risk in SCI. The longitudinal, international design of this project is considered a further strength; participants can be observed during the whole period of inpatient rehabilitation in different countries. Various factors like a central webbased database, a study nurse meeting, a comprehensive study manual and regular monitoring were implemented to ensure the standardisation of the measurements and data and thus to keep quality of the project in the 10 centres as high as possible.

For data collection, we used the International SCI Core Data Set, ${ }^{32}{ }^{33}$ the International SCI QoL Basic Data Set ${ }^{23}$ and the International SCI Pulmonary Function Data Set, ${ }^{22}$ which are developed to provide global data standards for SCI clinical research. The advantage of using these standardised data sets is to increase the data quality and to facilitate data sharing.

Potential limitations include the observational nature of the project which limits causal inference even within a prospective study design. Although the RESCOM sample reflects the composition of the source population quite well, we cannot exclude potential selection bias and, therefore, the results of the RESCOM project may not be transferable to all persons with SCI. There is a risk that the study inclusion and exclusion criteria may miss those patients with a potential high risk of pneumonia because those with more severe lesions or more complications may not consent to participate in an observational study that may not directly increase their outcome. Similarly, patients with a very long stay in the intensive care unit (eg, due to polytrauma) or a very late admission to the rehabilitation unit may miss T2 and, therefore, no more qualify for study participation. Those patients with 24 hours of mechanical ventilation are excluded since respiratory function cannot be measured and also those with other languages than used in our study centres are excluded. Another limitation of this project is that we do not have a follow-up period after the participants complete inpatient rehabilitation.

In summary, using discriminatory parameters of respiratory function, clinicians may identify persons with SCI who are at heightened risk of developing pneumonia during inpatient rehabilitation. Thus, interventions can be targeted at these persons to reduce pneumonia risk. The RESCOM study is well positioned to determine prognostic parameters of respiratory function for pneumonia risk in SCI.

\footnotetext{
Author affiliations

${ }^{1}$ Clinical Trial Unit, Swiss Paraplegic Center, Nottwil, Switzerland

${ }^{2}$ Life Course Epidemiology Group, Swiss Paraplegic Research, Nottwil, Switzerland

${ }^{3}$ Department of Respiratory and Sleep Medicine, Institute for Breathing and Sleep, Heidelberg, Victoria, Australia

${ }^{4}$ Department of Physiotherapy, University of Melbourne, Melbourne, Victoria, Australia
}

${ }^{5}$ Department of Rehabilitation Medicine, Rijndam Rehabilitation and Erasmus MC, University Medical Center Rotterdam, Rotterdam, The Netherlands

${ }^{6}$ Department of Rehabilitation Medicine, Heliomare Rehabilitation Center, Wijk aan Zee, The Netherlands

${ }^{7}$ Department of Spinal Cord Medicine, BG Trauma Hospital, Hamburg, Germany ${ }^{8}$ Center for Molecular Life Sciences, Radboud University Medical Center, Nijmegen, The Netherlands

${ }^{9}$ Trauma Surgery, AUVA Rehabilitation Center Häring, Bad Häring, Austria

${ }^{10} \mathrm{Clinic}$ for neurorehabilitation and paraplegiology, REHAB Basel, Basel, Switzerland

${ }^{11}$ Spinal Cord Unit, Clinique romande de réadaptation, Sion, Switzerland

${ }^{12}$ Spinal Cord Injury Center, Balgrist University Hospital, Zurich, Switzerland

${ }^{13}$ Allgemeine Unfallversicherungsanstalt, AUVA Rehabilitation Clinic Tobelbad, Tobelbad, Austria

\section{Twitter David Gobets @derdevid}

Acknowledgements The authors are grateful to all the participants of the RESpiratory COMplications study and all the study nurses for their great work in recruitment and data collection. The authors also acknowledge the financial support from Wings for Life (WFL-CH-014/16) and the Swiss Paraplegic Foundation for the SwiSCl nested project start-up grant.

Contributors GM is the principal investigator of the RESpiratory COMplications project. AMR and GM designed the study. MWGB provided statistical and methodological support. DJB, KP, DG, SH, MTEH, BH, MH-G, XJ, MS and RW provided clinical support and/or are responsible for data collection in their respective clinics. AMR, MWGB and GM drafted the work and DJB, KP, DG, SH, MTEH, BH, MH-G, XJ, MS and RW revised it critically for important intellectual content. AMR was responsible for the monitoring visits. All authors approved the final version of the manuscript and agreed to be accountable for all aspects of the work.

Funding This project is supported by Wings for Life, a spinal cord research foundation (Salzburg, Austria), grant number WFL-CH-014/16. Start-up costs for the participating Swiss Centres were covered by the SwiSCI nested project start-up grant of the Swiss Paraplegic Foundation.

Competing interests None declared.

Patient and public involvement Patients and/or the public were not involved in the design, or conduct, or reporting, or dissemination plans of this research.

Patient consent for publication Not required.

Ethics approval Approved by all local ethics committees of all participating centers, namely: for Switzerland: ethics committee north-west and central Switzerland (EKNZ) for the two Swiss Centres SPZ Nottwil and REHAB Basel, ethics committee Zurich, Switzerland for the Balgrist, Zurich and Ethics Committee Vaud, Switzerland for the CRR Sion (Nr. 2016-01065 - one multi-centric application for al Swiss centres). In Germany from the 'Ethikkommission der Ärztekammer Hamburg' (Nr. PV 5502); for Austria from the 'Ethikkommission der Medizinischen Universität Innsbruck' (Nr. AN-2016-0176) and the 'Ethikkommission für die Krankenanstalten der AUVA' (Nr. 6/2016). In the Netherlands from the medical ethics committee of the Erasmus Medical Centre Rotterdam (Nr. MEC-2016-594) and for Autralia from the Austin Health Human Research Ethics Committee (Nr. LNR/16/Austin/422).

Provenance and peer review Not commissioned; externally peer reviewed.

Open access This is an open access article distributed in accordance with the Creative Commons Attribution Non Commercial (CC BY-NC 4.0) license, which permits others to distribute, remix, adapt, build upon this work non-commercially, and license their derivative works on different terms, provided the original work is properly cited, appropriate credit is given, any changes made indicated, and the use is non-commercial. See: http://creativecommons.org/licenses/by-nc/4.0/.

\section{ORCID iDs}

Anja M Raab http://orcid.org/0000-0002-4139-2173

Martin W G Brinkhof http://orcid.org/0000-0002-9319-665X

David J Berlowitz http://orcid.org/0000-0003-2543-8722

Karin Postma http://orcid.org/0000-0002-0729-6778

David Gobets http://orcid.org/0000-0003-0270-5672

Maria T E Hopman http://orcid.org/0000-0001-9504-5452

Margret Hund-Georgiadis http://orcid.org/0000-0002-4415-2888

Xavier Jordan http://orcid.org/0000-0003-3389-0057

Renate Wildburger http://orcid.org/0000-0001-9820-3823

Gabi Mueller http://orcid.org/0000-0001-6391-3737 


\section{REFERENCES}

1 Schilero GJ, Radulovic M, Wecht JM, et al. A center's experience: pulmonary function in spinal cord injury. Lung 2014;192:339-46.

2 Chamberlain JD, Buzzell A, Gmünder HP, et al. Comparison of allcause and cause-specific mortality of persons with traumatic spinal cord injuries to the general Swiss population: results from a national cohort study. Neuroepidemiology 2019;52:205-13.

3 Cardenas DD, Hoffman JM, Kirshblum S, et al. Etiology and incidence of rehospitalization after traumatic spinal cord injury: a multicenter analysis. Arch Phys Med Rehabil 2004;85:1757-63.

4 Dryden DM, Saunders LD, Rowe BH, et al. Utilization of health services following spinal cord injury: a 6-year follow-up study. Spinal Cord 2004;42:513-25.

5 Aarabi B, Harrop JS, Tator $\mathrm{CH}$, et al. Predictors of pulmonary complications in blunt traumatic spinal cord injury. J Neurosurg Spine 2012;17:38-45.

6 Croce MA, Fabian TC, Waddle-Smith L, et al. Identification of early predictors for post-traumatic pneumonia. Am Surg 2001;67:105-10.

7 Postma K, Bussmann JB, Haisma JA, et al. Predicting respiratory infection one year after inpatient rehabilitation with pulmonary function measured at discharge in persons with spinal cord injury. $J$ Rehabil Med 2009;41:729-33.

8 Agostinello J, Battistuzzo CR, Batchelor PE. Early clinical predictors of pneumonia in critically ill spinal cord injured individuals: a retrospective cohort study. Spinal Cord 2019;57:41-8.

9 Jaja BNR, Jiang F, Badhiwala $\mathrm{JH}$, et al. Association of pneumonia, wound infection, and sepsis with clinical outcomes after acute traumatic spinal cord injury. J Neurotrauma 2019;36:3044-50.

10 Berlowitz DJ, Tamplin J, Cochrane Injuries Group. Respiratory muscle training for cervical spinal cord injury. Cochrane Database Syst Rev 2013;72:CD008507.

11 Boswell-Ruys CL, Lewis CRH, Wijeysuriya NS, et al. Impact of respiratory muscle training on respiratory muscle strength, respiratory function and quality of life in individuals with tetraplegia: a randomised clinical trial. Thorax 2020;75:279-88.

12 van Silfhout L, Peters AEJ, Berlowitz DJ, et al. Long-Term change in respiratory function following spinal cord injury. Spinal Cord 2016;54:714-9.

13 Bican O, Minagar A, Pruitt AA. The spinal cord: a review of functional neuroanatomy. Neurol Clin 2013;31:1-18

14 Lanig IS, Peterson WP. The respiratory system in spinal cord injury. Phys Med Rehabil Clin N Am 2000;11:29-43.

15 Kang SW, Shin JC, Park Cl, et al. Relationship between inspiratory muscle strength and cough capacity in cervical spinal cord injured patients. Spinal Cord 2006;44:242-8.

16 Kang SW, Bach JR. Maximum insufflation capacity: vital capacity and cough flows in neuromuscular disease. Am J Phys Med Rehabil 2000;79:222-7.
17 Park JH, Kang S-W, Lee SC, et al. How respiratory muscle strength correlates with cough capacity in patients with respiratory muscle weakness. Yonsei Med J 2010;51:392-7.

18 Postma K, Vlemmix LY, Haisma JA, et al. Longitudinal association between respiratory muscle strength and cough capacity in persons with spinal cord injury: an explorative analysis of data from a randomized controlled trial. J Rehabil Med 2015;47:722-6.

19 Kirshblum SC, Waring W, Biering-Sorensen F, et al. Reference for the 2011 revision of the International standards for neurological classification of spinal cord injury. J Spinal Cord Med 2011;34:547-54.

20 Post MWM, Brinkhof MWG, von Elm E, et al. Design of the Swiss spinal cord injury cohort study. Am J Phys Med Rehabil 2011;90:S5-16.

21 Horan TC, Andrus M, Dudeck MA. CDC/NHSN surveillance definition of health care-associated infection and criteria for specific types of infections in the acute care setting. Am J Infect Control 2008;36:309-32.

22 Biering-Sørensen F, Krassioukov A, Alexander MS, et al. International spinal cord injury pulmonary function basic data set. Spinal Cord 2012;50:418-21.

23 Charlifue S, Post MW, Biering-Sørensen F, et al. International spinal cord injury quality of life basic data set. Spinal Cord 2012;50:672-5.

24 American Thoracic Society. Standardization of spirometry, 1994 update. Am J Respir Crit Care Med 1995;152:1107-36.

25 Miller MR, Hankinson J, Brusasco V, et al. Standardisation of spirometry. Eur Respir J 2005;26:319-38.

26 American Thoracic Society/European Respiratory Society. ATS/ERS statement on respiratory muscle testing. Am J Respir Crit Care Med 2002;166:518-624.

27 Wadsworth BM, Haines TP, Cornwell PL, et al. Abdominal binder use in people with spinal cord injuries: a systematic review and metaanalysis. Spinal Cord 2009;47:274-85.

28 Hyppönen E, Berry DJ, Wjst M, et al. Serum 25-hydroxyvitamin $\mathrm{D}$ and $\lg \mathrm{E}$ - a significant but nonlinear relationship. Allergy 2009;64:613-20.

29 Verkuilen J. Explanatory item response models: a generalized linear and nonlinear approach by P. de Boeck and M. Wilson and generalized latent variable modeling: multilevel, longitudinal and structural equation models by A. Skrondal and S. Rabe-Hesketh. Psychometrika 2006;71:415-8.

30 Steyerberg EW, Moons KGM, van der Windt DA, et al. Prognosis research strategy (progress) 3: prognostic model research. PLoS Med 2013;10:e1001381.

31 Altman DG, Vergouwe $\mathrm{Y}$, Royston $\mathrm{P}$, et al. Prognosis and prognostic research: validating a prognostic model. BMJ 2009;338:b605.

32 Biering-Sørensen F, Charlifue S, DeVivo M, et al. International spinal cord injury data sets. Spinal Cord 2006;44:530-4.

33 Biering-Sørensen F, Charlifue S, Devivo MJ, et al. Using the spinal cord injury common data elements. Top Spinal Cord Inj Rehabil 2012;18:23-7. 